\title{
Spacial Flexible cable Dynamics Modeling and Finite Element Analysis Based on Absolute Nodal Coordinate
}

\author{
MA Chao ${ }^{1, *}$, WANG Ran ${ }^{1}$, WEI Cheng ${ }^{1}$, ZHAO Yang $^{1}$ \\ ${ }^{1}$ Dept. of Aerospace Engineering, Harbin Institute of Technology, Harbin, 150001, China
}

Keywords: Absolute Nodal Coordinate; Flexible cable; Dynamics Modeling; Finite Element Analysis

Abstract. First this paper expresses kinematics relationships of Plane clearance revolute air and spatial clearance spherical hinge using absolute nodal coordinate method. This paper introduces a computation model of continuous normal collision force which can consider dissipation of collision energy. In the meantime, in order to avoid numerical value problems, this paper adopts an improved friction mode to calculate the friction force during collision. It further deducts the friction force moment computation formula basing on absolute nodal coordinate and establishes multibody dynamics model with clearance kinematic pair which considers friction effects.

\section{Introduction}

ANCF cable-beam element adopts Green strain to describe large displacement, large rotation and large strain problems directly basing on basic theories of continuum mechanics which solves the limitation of small deformation and small corners of traditional finite elements and can reduce the amount of elements greatly under the premise of guaranteeing accuracy and enhance the computation efficiency of orbital net. In the meantime, it can also select to consider axial direction, bending, cutting and torsional deformation according to the computation efficiency of orbital net and accuracy requirement as well as give kinetic energy and potential energy under any status of flexible cable element to further describe the constitutive mechanical model of cables accurately. It can also provide accurate models to model correction of ground test which can provide accurate analysis elements to orbital net model. The modeling process of flexible element is shown as follows:

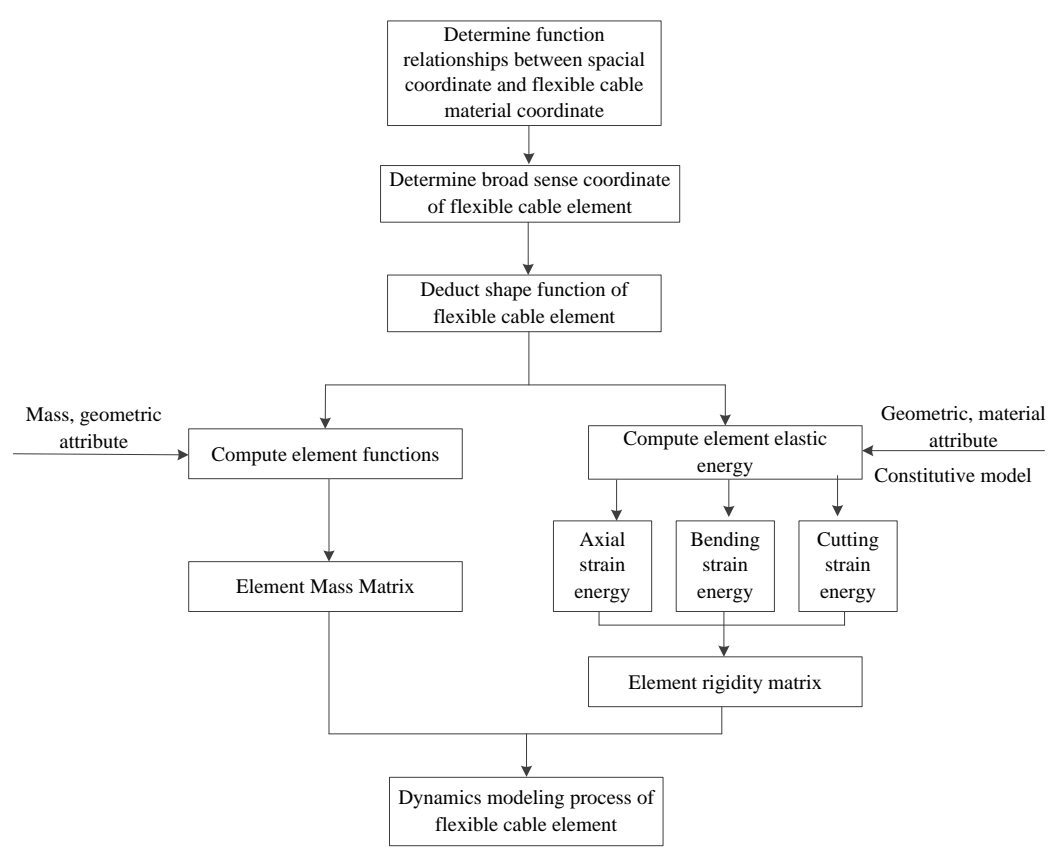

Fig.1. Dynamics modeling process of flexible cable element 


\section{Cable Nodes and Their Connecting Dynamics Attribute Modeling}

The orbital net is connected by large-scale cables through connecting nodes. The dynamics characteristics of qualities and rigidity of cable nodes are completely different from cables. Their connecting rigidity shows a high non-linearity and its connecting damping are much more complicated which can not be treated with the simple connection of cables and mass piece rigidity. The non-linear connection attribute at connecting nodes will also seriously influence the dynamic attribute of the whole orbital net. So it needs to carry out modeling study of dynamics attribute of connecting nodes and implement corresponding experiment and verification to dynamics model basing on this.

The orbital net can be express as the basic element shown in Figure 2(a) which includes flexible cable element and cable connecting nodes. The cable nodes are approximately expressed as concentrated mass element and also connect to the surrounding flexible cable element which have a complex connecting property with high non-linearity and can be approximately expressed as non-linear spring damping connection (As shown in Figure 2 (b)). In the Figure, 0 expresses the connecting nodes and 1-4 express the flexible cable ends connecting with the nodes. $\mathrm{r} 0$ and ri ( $i=1,2,3,4)$ indicate the position vectors of cable nodes and flexible ends respectively under inertial system.

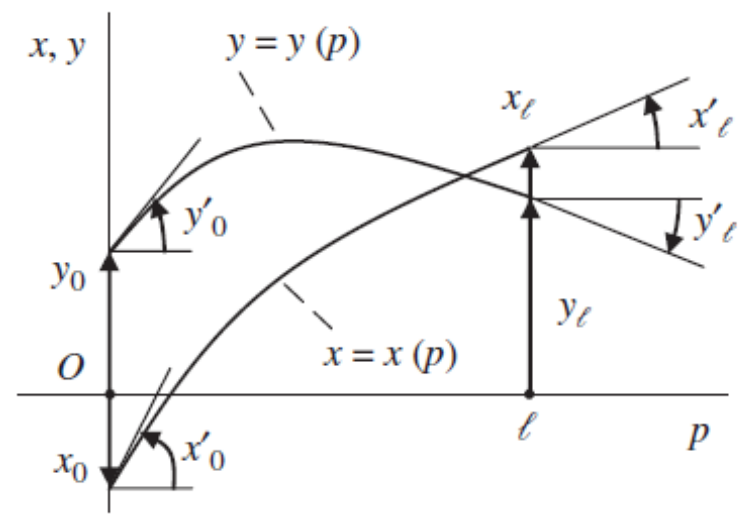

Fig.2. Parameterization of beam.

The key point of cable nodes and its connecting property dynamics modeling is to calculate the non-linear connection at the node which can be expressed as follows:

$$
\begin{aligned}
\mathrm{T}_{i} & =K_{i} \delta_{i}+b_{i} \dot{\delta}_{i} \quad i=1,2,3,4 \\
\delta_{i} & =r_{0}-r_{i}
\end{aligned}
$$

The $K$ and $b$ are non-linear rigidity coefficient and damping coefficient respectively whose specific values need to be corrected through corresponding cable constitutive mechanics model experiment.

During the process of orbital net capturing spacecraft, the cable net will have self collision. So the contact collision inspection and collision force of flexible cables themselves need to be calculated. In the meantime, because the surface of flexible cables is covered with fiber burrs and so on, the parameters such as contact rigidity, damping coefficient, friction coefficient have great differences with traditional rigid hertz contact collision force. So corresponding exquisite collision dynamics modeling and simplified dynamics equivalent model should be implemented and corresponding experiment and verification should be carried out basing on this.

The inspection and calculation process of flexible cables from contact collision: 


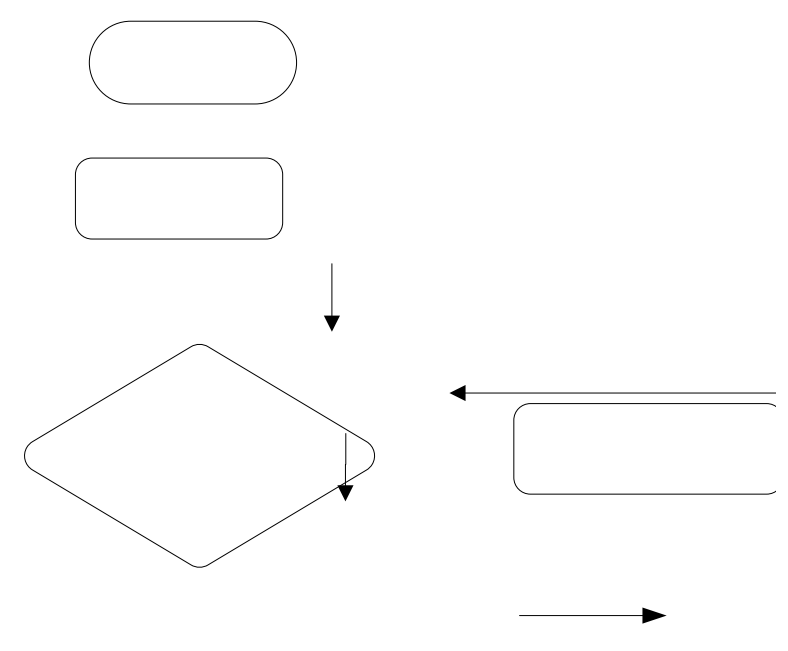

Fig.3. The inspection and calculation process of flexible cables from contact collision

First inspect the relative locations of different flexible cables through point-to-point inspection algorithm. If there is mutual invasion among cables, calculate the invasion amount among cables and then further calculate corresponding contact collision force basing on the moment flexible cables contact collision model and update moving state of flexible cables. Repeat the above process in the next step calculation.

For the inspection of self-collision of flexible cables, adopt absolute nodal coordinate finite element collision inspection method and point-to-point inspection method and judge whether there is contact between two cables through calculation of distance among cables. The calculation of flexible cable from contact collision force is the same with the previous section which also include normal collision force and tangential friction force which will not be repeated here. The parameters such as rigidity coefficient, damping coefficient, friction coefficient needed by the calculation of contact force among cables depend on the geometric and material properties of cables. First inspect moving bodies through point-to-face inspection algorithm. If there is invasion among moving bodies, then calculate the invasion amount of target geometry to flexible cables and then further calculate contact collision according to contact mode between flexible model and target and repeat the whole process in the next step calculation under the state of updated moving bodies.

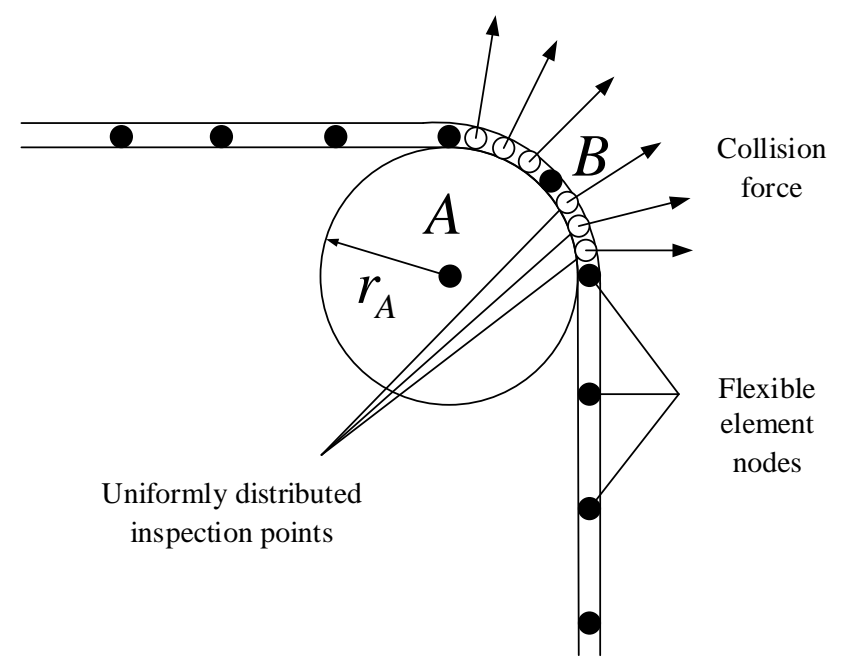

Fig.4. Inspection schematic diagrams of point-to-sphere

When inspecting whether there is collision among flexible cables elements, adopt point and face inspections of absolute nodal finite element collision inspection method and judge whether this inspection point invades inspection face through calculating the distance from inspection point to all inspection faces.

$C_{A}$ and $C_{B}$ indicate sphere center coordinate and certain inspection point coordinate on the 
element respectively. $r_{A}$ is the radius of this sphere. When the distance from point to sphere is smaller than the radius of this sphere, then there is contact between point and face:

$$
\left|\vec{c}_{A}-\vec{c}_{B}\right|<r_{A}
$$

The position of collision point is the position of inspection point $C_{A}$. The normal direction of collision can be calculated by the following formula:

$$
\vec{n}=\frac{\vec{c}_{B}-\vec{c}_{A}}{\left|\vec{c}_{B}-\vec{c}_{A}\right|}
$$

The collision invasion amount from point to sphere face is:

$$
d=r_{A}-\left|\vec{c}_{A}-\vec{c}_{B}\right|
$$

For on-dimensional flexible cable elements, because the absolute nodal coordinate element grid is sparse, so arranging several inspection points inside the element is needed. These inspection points can be distributed inside the element evenly or can be gauss integral points inside the element.

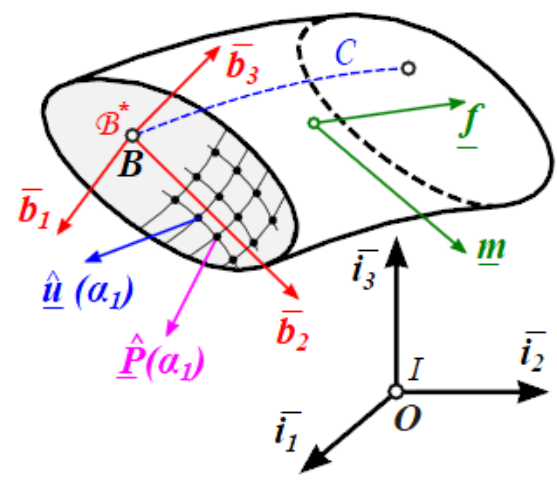

Fig.5. Geometric inspection body in flexible element

For one-dimensional flexible element, the geometric inspection body of the element can be composed of several cylinders according to cross-section shape of the element. Determine the number of geometries inside one element according to specific model. The radius of cylinder is determined by cross-section size of flexible cable element and the generatrix length of cylinder is determined by axial size of flexible cable and number of geometries inside the element. So in collision inspection process, the geometric size of cable is really considered. The flexible cable collision geometry established with this method can be carried out collision inspection with geometries of any CAD shapes.

For the calculation of collision force, use virtual work principle that the contact force acting on element can be transferred to element node broad sense force $Q_{c}^{f}$ :

$$
Q_{c}^{f}=N(p)^{T} f
$$

The $\mathrm{p}$ is element coordinate parameter corresponding to element collision points which is determined by collision point $r_{p} . f=f_{n} n+f_{t} t$ is the contact force on the collision point which include normal collision force $f_{n}$ and friction force $f_{t}$. The $\mathrm{n}$ and $\mathrm{t}$ is collision normal unit vector and tangential unit vector respectively.

\section{Experiment and Analysis}

Through overall consideration of direction locations tracing errors of $\mathrm{X}, \mathrm{Y}$, absolute nodal coordinate method and hypothesis modal method, it is obtained that the position tracking results of accuracy of $\mathrm{P}$ point are similar. Figure 7 shows that the track obtained from hypothesis modal method coincides with the track needs fairly. 


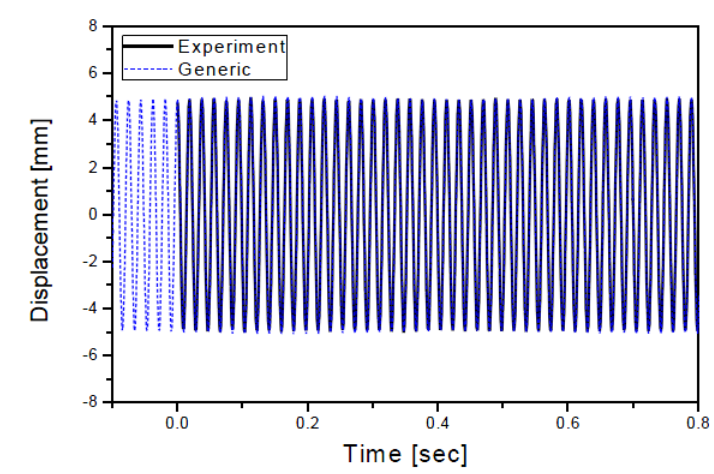

(a) Steady state response

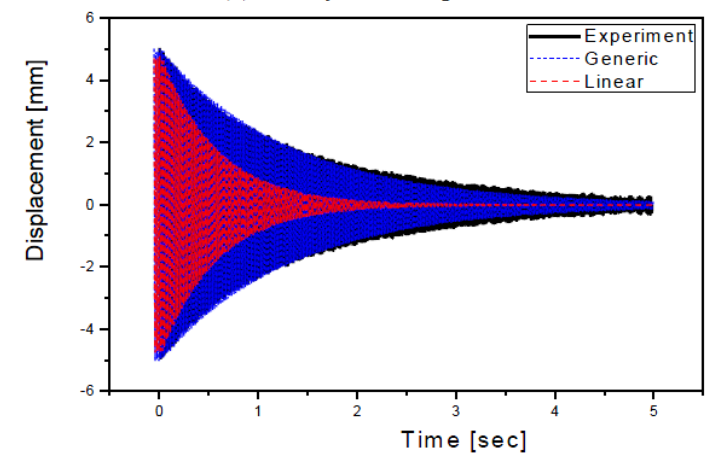

(b) Transient decay response

Fig.6. Comparison of experiment and simulation

One can understand that the damping ratios of the linear damping model are higher than those of physical experiment above the 3rd mode. Especially, the 5th damping ratio is different by about $57 \%$. Contrary to the linear damping model, the generic damping model can estimate the damping characteristics well enough in spite of the high frequency region because it directly uses the modal parameters of each mode achieved from experiment. If one can measure the modal parameters of the higher frequency, the generic damping model can control all frequency behavior which can be calculated. Until now, it has been shown that the linear damping model cannot predict the response in high order behavior and the generic damping model which has the frequency dependent attribute can predict the response well enough in spite of high frequencies.

\section{Conclusion}

The study presents the proper correlation method with introducing the frequency dependent generic damping model. This research shows with the linear damping model, which is used traditionally for simplicity, it is impossible to express the high frequency motion at the same time, and the proposed generic damping model can suppress the number of frequency modes simultaneously. The absolute nodal coordinate formulation uses the global displacement and finite slope as nodal coordinates. So, this paper presents interesting results that the traditional modal damping theory can also be used even in the case of the nodal coordinates consisting of global displacement and finite slope. This paper shows that the modal transformation technique can be also applicable to a beam undergoing large displacements. Finally, through comparisons between numerical simulation and experiment, this study shows that the presented generic damping matrix, modal testing and parameter identification method works well in flexible multibody dynamics undergoing large displacement.

\section{References}

[1] Jie He, Yishuang Geng and Kaveh Pahlavan, Toward Accurate Human Tracking: Modelling Time-of-Arrival for Wireless Wearable Sensors in Multipath Environment, IEEE Sensor Journal, 14(11), 3996-4006, Nov. 2014

[2] Yishuang Geng, Kaveh Pahlavan, On the Accuracy of RF and Image Processing Based Hybrid 
Localization for Wireless Capsule Endoscopy, IEEE Wireless Communications and Networking Conference (WCNC), Mar. 2015

[3] Jie He, Yishuang Geng and Kaveh Pahlavan, Toward Accurate Human Tracking: Modelling Time-of-Arrival for Wireless Wearable Sensors in Multipath Environment, IEEE Sensor Journal, 14(11), 3996-4006, Nov. 2014

[4] Lv, Zhihan, and Tianyun Su. "3D seabed modeling and visualization on ubiquitous context." In SIGGRAPH Asia 2014 Posters, p. 33. ACM, 2014.

[5]Wenhua Huang, Yishuang Geng, Identification Method of Attack Path Based on Immune Intrusion Detection, Journal of Networks, 9(4), 964-971, Jan. 2014 